This item was submitted to Loughborough's Research Repository by the author.

Items in Figshare are protected by copyright, with all rights reserved, unless otherwise indicated.

\title{
Modeling diffusion directions of Corpus Callosum
}

PLEASE CITE THE PUBLISHED VERSION

https://doi.org/10.1007/978-3-319-60964-5_45

PUBLISHER

(C) Springer Verlag (Germany)

\section{VERSION}

AM (Accepted Manuscript)

\section{PUBLISHER STATEMENT}

This work is made available according to the conditions of the Creative Commons Attribution-NonCommercialNoDerivatives 4.0 International (CC BY-NC-ND 4.0) licence. Full details of this licence are available at: https://creativecommons.org/licenses/by-nc-nd/4.0/

\section{LICENCE}

CC BY-NC-ND 4.0

\section{REPOSITORY RECORD}

Elsheikh, Safa, Andrew Fish, Roma Chakrabarti, Diwei Zhou, and Mara Cercignani. 2019. "Modeling Diffusion Directions of Corpus Callosum". figshare. https://hdl.handle.net/2134/25002. 


\title{
Modeling Diffusion Directions of Corpus Callosum
}

\author{
Safa Elsheikh ${ }^{1}$, Andrew Fish ${ }^{1}$, Roma Chakrabarti ${ }^{1}$, Diwei Zhou ${ }^{2 \star}$, and Mara \\ Cercignani $^{3,4}$ \\ ${ }^{1}$ School of Computing, Engineering and Mathematics, University of Brighton, \\ United Kingdom \\ 2 Department of Mathematical Sciences, Loughborough University, \\ United Kingdom \\ 3 Clinical Imaging Sciences Centre, Department of Neuroscience, Brighton and \\ Sussex Medical School, University of Sussex, United Kingdom \\ 4 Neuroimaging Laboratory, Santa Lucia Foundation, Rome, Italy
}

\begin{abstract}
Diffusion Tensor Imaging (DTI) has been used to study the characteristics of Multiple Sclerosis (MS) in the brain. The von MisesFisher distribution (vmf) is a probability distribution for modeling directional data on the unit hypersphere. In this paper we modeled the diffusion directions of the Corpus Callosum (CC) as a mixture of vmf distributions for both MS subjects and healthy controls. Higher diffusion concentration around the mean directions and smaller sum of angles between the mean directions are observed on the normal-appearing $\mathrm{CC}$ of the MS subjects as compared to the healthy controls.
\end{abstract}

Keywords: Diffusion Tensor Imaging, Multiple Sclerosis, von MisesFisher, Corpus Callosum, concentration, mean directions.

\section{Introduction}

The von Mises-Fisher distribution (vmf) is one of the most basic probability distributions for modeling directional data on the unit hypersphere [1]. The use of a finite mixture of vmf distributions to cluster directional data on the unit sphere has been proposed by [2]. They used an Expectation Maximization (EM) algorithm to estimate the parameters of the mixture model. Much research has been performed to model the diffusion imaging of the brain as a vmf distribution. For example, [3] model the orientation distribution function (ODF) of High Angular Resolution Diffusion Imaging (HARDI) as a mixture of vmf distributions. They also use this model for segmentation using synthetic and real HARDI data. In [4], they proposed a $5 D$ hyper spherical model for HARDI data using a mixture of vmf distributions. A method for reconstructing Diffusion Weighted Magnetic Resonance (DW-MR) signal using a continuous mixture of vmf distributions has been introduced in [5]. They validate the method using synthetic

\footnotetext{
* corresponding author
} 
and real brain data. A probabilistic fiber tracking algorithm using a particle filtering technique and vmf sampling has been proposed in [6]. In [7], they build a model of glioma growth using diffusion tensor imaging (DTI) and a bimodal vmf distribution. The vmf distribution, spherical harmonic (SH) expansion and Fractional anisotropy (FA) have been used in [8] for the classification of Alzheimer's disease. Comparing the results, they found that vmf and $\mathrm{SH}$ outperform the FA in the classification.

Multiple Sclerosis (MS) is an immune-mediated neurological disease, characterized by recurring inflammatory events (relapsing-remitting MS), associated with demyelinating lesions within the white matter of the brain and spinal cord. The majority of patients after a variable period of relapsing-remitting course develop the so-called secondary-progressive form of MS, characterised by a chronic accumulation of disability, with less and less acute events. Microscopic damage is known to occur also outside of macroscopic lesions, and DTI was found to be sensitive to such abnormalities e.g. [9-14]. The Corpus Callosum (CC) is the largest bundle of commissural fibers in the brain, and damage to it has been associated with increased risk of developing disability in MS. The FA of the CC has been reported to be decreased and the Mean Diffusivity to be increased for MS subjects.

In this paper, we modeled the diffusion directions in the $\mathrm{CC}$ as a mixture of vmf distributions. This allows us to determine the distinct mean diffusion directions in the CC. Also it helps to cluster the CC depending on the diffusion directionality. We study the diffusion directions in the whole normal-appearing $\mathrm{CC}$ and the lesions are not included. We refer to normal-appearing CC briefly as CC. We briefly review single and finite mixtures of vmf distributions in Sect. 2. In Sect. 3, we directly apply the mixture of vmf to model the CC directions for healthy controls and MS patients. We find that there are at least three distinct mean directions in the three dimensional CC. The results are presented in Sect. 4. The diffusion concentrations around the mean directions are higher for MS subjects. Moreover, the sum of angles between the three mean directions are smaller for MS subjects. We conclude with a brief summary and future work in Sect. 5 .

\section{Von Mises-Fisher Distribution}

The vmf is a probability distribution on the $(d-1)$ dimensional sphere in $R^{d}$. The vmf density function of the unit vector $\mathbf{x}$, given the mean direction $\boldsymbol{\mu}$ and the concentration around the mean $K$, is given by:

$$
f(\mathbf{x} \mid \boldsymbol{\mu}, K)=c_{d}(K) e^{K \boldsymbol{\mu}^{T} \mathbf{x}},
$$

where $\|\boldsymbol{\mu}\|=1$ and $K>0$. The normalizing constant $c_{d}(K)$ is given by:

$$
c_{d}(K)=\frac{K^{d / 2-1}}{(2 \pi)^{d / 2} I_{d / 2-1}(K)},
$$


where $I_{v}($.$) is the modified Bessel function of the first kind at order v$. If $d=2$ this distribution is von Mises distribution on the circle, which is the circular analogue of the normal distribution. If $d=3$ the normalization constant can be written as

$$
c_{3}(K)=\frac{K}{4 \pi \sinh (K)} .
$$

The density of finite mixtures $H$ of vmf distributions is given by

$$
p(\mathbf{x} \mid \Theta)=\sum_{h=1}^{H} w_{h} f\left(\mathbf{x} \mid \theta_{h}\right),
$$

where $\Theta=\left\{w_{1}, w_{2}, \ldots, w_{H}, \theta_{1}, \theta_{2}, \ldots, \theta_{H}\right\}$ are the parameters of the mixture density and $f\left(\mathbf{x} \mid \theta_{h}\right)$ is the density of the vmf distribution with parameter $\theta_{h}=$ $\left\{\boldsymbol{\mu}_{h}, K_{h}\right\}$. The water diffusion in the white matter in the brain is parallel to the principal eigenvector of the tensor. In the following Sect., we modeled the principal eigenvectors of the tensors in the $\mathrm{CC}$ as a mixture of vmf distributions to study the means and the concentrations of water diffusion in the CC.

\section{Modeling the Diffusion Direction of the Corpus Callosum}

The data was collected at the Neuroimaging Laboratory, Santa Lucia Foundation in Rome (Italy). The study was approved by Ethics Committee of Santa Lucia Foundation. Written informed consent was obtained from all subjects before entering the study. The data consists of four healthy and nine secondary progressive MS subjects. Diffusion-weighted imaging was obtained using a headonly 3.0 T scanner (Siemens Magnetom Allegra, Siemens Medical Solutions, Erlangen, Germany), using a twice-refocused spin echo echo-planar imaging (SE $\mathrm{EPI})$ sequence $\left(\mathrm{TR}=7000 \mathrm{~ms}, \mathrm{TE}=85 \mathrm{~ms}\right.$, maximum $\mathrm{b}$ factor $=1000 \mathrm{~s} / \mathrm{mm}^{2}$, isotropic resolution $2.3 \mathrm{~mm}^{3}$; matrix $=96 \times 96$; 60 slices $)$, accomplished by collecting 7 images with no diffusion weighting $(b=0)$ and 61 images with diffusion gradients, applied in 61 non-collinear directions (scan time: $11 \mathrm{~min}$ ).

The three dimensional CC is segmented using the Euclidean method discussed in [15]. The principal eigenvectors of the diffusion tensors in the CC are parallel to the diffusion directions in the CC. Hence, the unit vector $\mathbf{x}$ represents the principal eigenvector, $\boldsymbol{\mu}$ is the mean diffusion direction and $K$ is the concentration of the diffusion directions around $\boldsymbol{\mu}$. Intuitively, the concentration is the opposite of the variation and spread. As the diffusion tensor is a $3 \times 3$ matrix, the principal eigenvector $\mathbf{x}$ of the tensor is a $3 \times 1$ vector so $d=3$. Substituting (1) and (3) into (4) gives:

$$
p(\mathbf{x} \mid \Theta)=\frac{1}{4 \pi} \sum_{h=1}^{H}\left[\frac{w_{h} K_{h}}{\sinh \left(K_{h}\right)} e^{K_{h} \boldsymbol{\mu}_{h}^{T} \mathbf{x}}\right],
$$


Fitting the mixture of vmf distributions has been performed using the Expectation Maximization (EM) [2]. For implementation of the EM algorithm using $\mathrm{R}$ language see [16]. The Bayesian information criterion (BIC) can be used for model selection to choose the most suitable model. The model with the smallest BIC is the preferred model. The BIC is defined as:

$$
B I C=h \log (n)-2 \log (L),
$$

where $h$ is the number of parameters to be estimated, $n$ is the sample size and $L$ is the maximized likelihood function of the model. The BIC values showed that a mixture of vmf distributions for the $\mathrm{CC}$ direction is preferred over a single vmf distribution for both healthy and MS patients (see Fig. 1(b) and 1(a)). From the figures, it is clear that the BIC values for three mixture distributions are lower than one and two mixture distributions in all cases for both the MS and healthy controls. Thus, at least three mixtures of vmf distributions are preferred, to model the diffusion in the whole CC. The BIC values for four and five mixture distributions are either bigger or slightly smaller than BIC values for three mixture distributions. Hence, to be able to compare the models for all the subjects with the same number of mixture components, we choose to model the data using three mixtures of vmf distributions (i.e. $H=3$ in (5)). The mixture distributions of the CC for one healthy control and one MS subject are shown in Fig. 2.

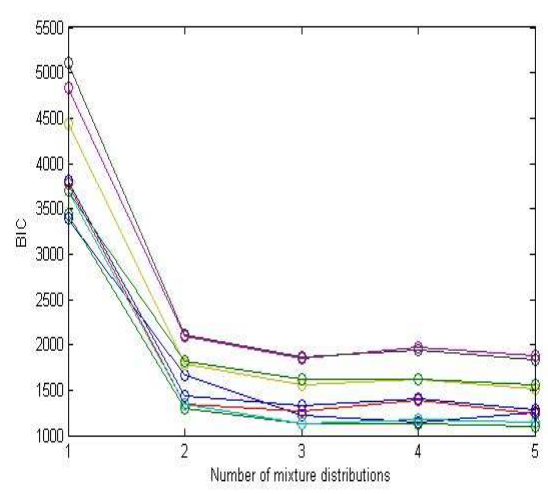

(a) BIC for the nine MS subjects.

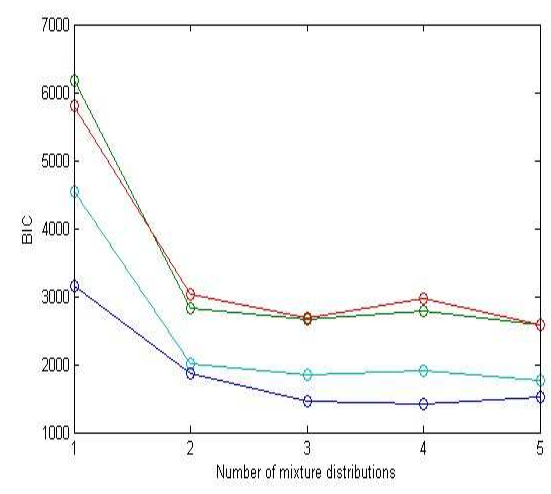

(b) BIC for the four healthy controls.

Fig. 1: Bayesian information criterion (BIC) for MS subjects and healthy controls. 


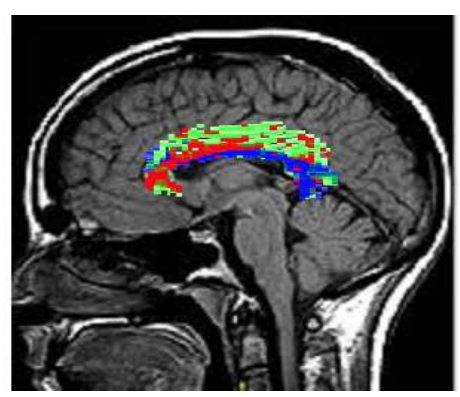

(a) The mixture directions for a MS subject.

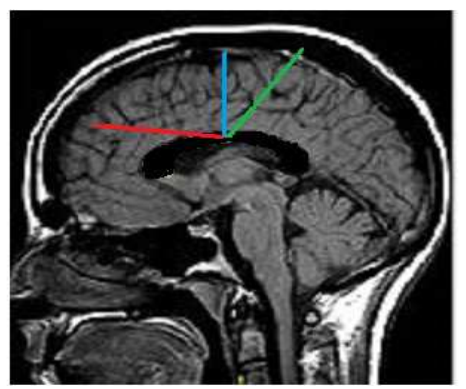

(c) The mean directions for the three mixture distributions in (a). The sum of angles between each two of the mean directions is 77.99 .

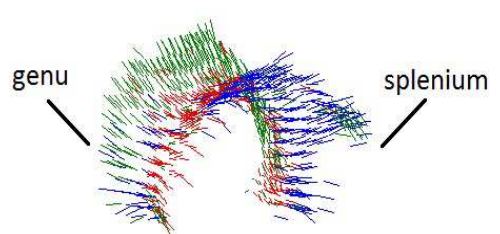

(e) The $3 D$ view of the mixture directions in (a). The concentration values for the regions (blue, green, red) are $(23.36,13.48,20)$.

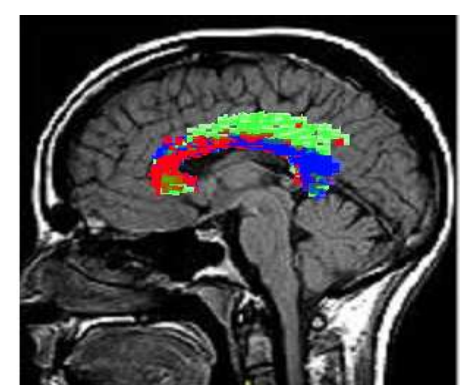

(b) The mixture directions for a healthy control.

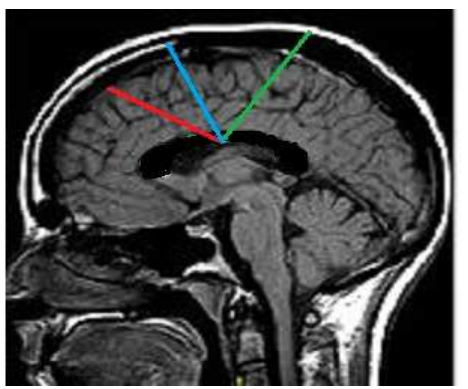

(d) The mean vectors for the three mixture distributions in (b). The sum of angles between each two of the mean directions is 83.97.

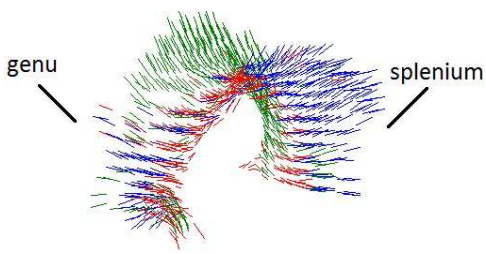

(f) The $3 D$ view of the mixture directions in (b). The concentration values for the regions (blue, green, red) are $(18.21,12.02,14.29)$.

Fig. 2: The CC is clustered into three regions (blue, green and red) using the mixture of vmf distributions for a MS subject and a healthy control. The sum of angles between each of the three mean directions are smaller for the MS subject. For both the MS subject and healthy control, the diffusion directions on the blue and red regions are more concentrated than on the green region. The diffusion concentrations around the mean directions for MS subject are higher than for the healthy control. 


\section{Results}

Although the average of the FA over the whole CC is higher for the healthy controls than for the MS subjects (Fig. 3(a)), the difference is not significant $(p$-value $=0.604)$. However, there is a significant difference in the FA variation $(\mathrm{p}$-value $=0.000)$ in the CC between the MS and healthy control (Fig. 3(b)). To compare the diffusion direction concentration between MS subjects and healthy controls, we need to model the data (the principal eigenvectors of the tensors in the $\mathrm{CC}$ ) as vmf distributions. The BIC values show that there are at least three different diffusion directions, so we modeled the data as a mixture of three vmf distributions. We calculate the angles between each two of the three mean directions. As the diffusion is a symmetric process (we cannot distinguish between left to right diffusion and right to left, this means the angle 0 is equivalent to the angle $\pi$ ), the angle is calculated as the minimum of $\theta$ and $(\pi-\theta)$ where $\theta$ is the angle between two mean vectors and thus we get angles between 0 and $\pi / 2$. We find that the sum of the angles between each two of the three mean directions are significantly higher for healthy controls than MS subjects (p-value $=0.007)$. This result shown in Fig. 4(a). Then we compare the concentration values between MS subjects and the healthy controls (Fig. 4(b)). The diffusion concentrations of MS subjects are significantly higher than the diffusion concentrations of the healthy controls ( $\mathrm{p}$-value $=0.001$ ). These might be a result of the atrophy in the CC of MS subjects which leads to decrease the spread of the diffusion directions over the CC.

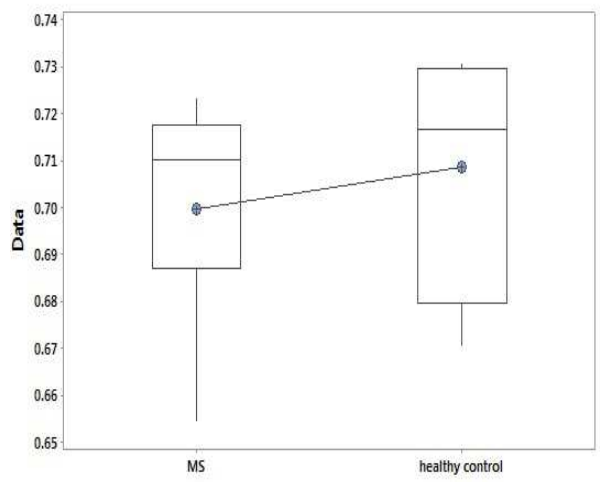

(a) Boxplots of means of FA of the CC.

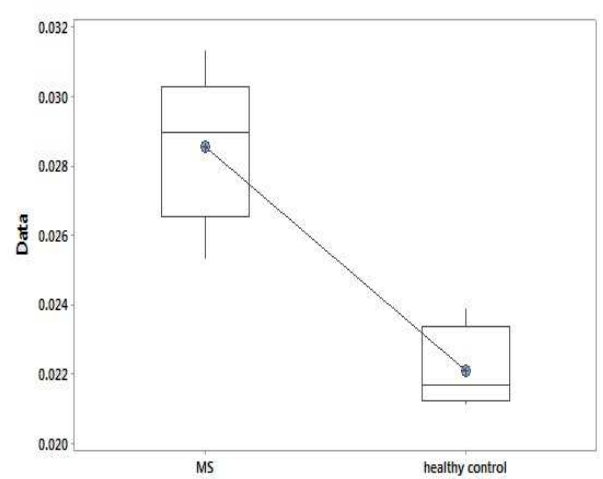

(b) Boxplots of variances of FA of the CC.

Fig. 3: Boxplots of means and variances of FA for MS subjects and healthy controls. 


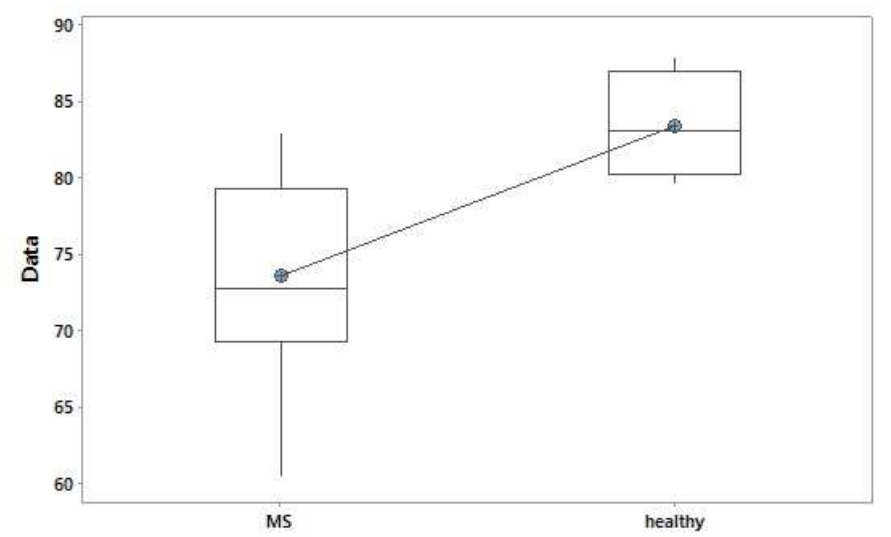

(a) Sum of angles between the three mean directions for MS subjects and healthy controls.

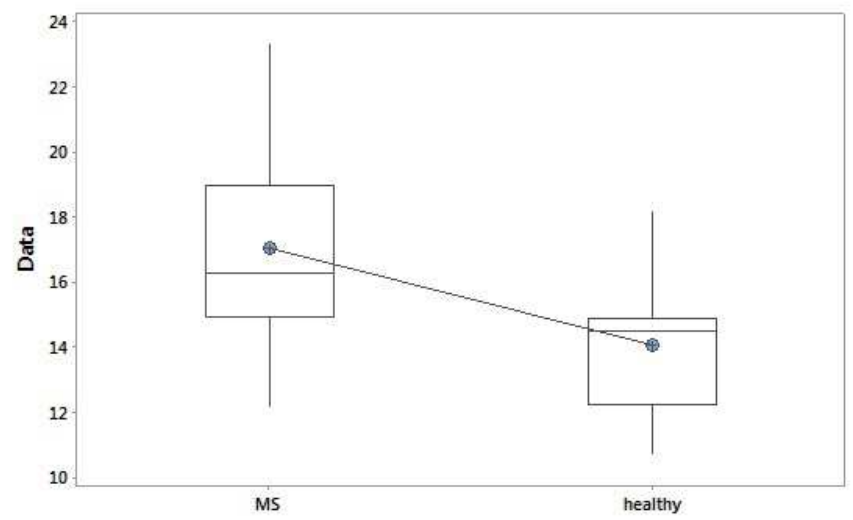

(b) Diffusion concentrations for MS subjects and healthy controls.

Fig. 4: Boxplots of concentrations and sums of angles between the three mean directions. 


\section{Conclusion}

We have used a mixture of vmf distributions to model the diffusion in the three dimensional CC. There are at least three different mean diffusion directions in the CC. On the normal-appearing CC of MS subjects the sum of angles between the mean diffusion directions are smaller with higher concentration around the mean directions compared with the healthy controls. This due to the atrophy in the $\mathrm{CC}$ of the MS subjects. Future work will address how these results will be affected when the regions with MS lesions in the $\mathrm{CC}$ are included in the analysis. The result in this paper used the Euclidean method for segmenting the $\mathrm{CC}$ as it is faster than the segmentation methods using non-Euclidean measures but it is less accurate than other non-Euclidean methods [15]. Future work will also include using the segmentation results obtained by non-Euclidean methods and by other segmentation methods to investigate more about the consistency of the results in this paper when using different segmentation methods. Using the vmf to obtain similar results as Witelson subdivisions of the CC [17] is also of interest. Furthermore, a larger data set will be considered.

\section{References}

[1] Kanti V Mardia and Peter E Jupp. Directional statistics, volume 494. John Wiley \& Sons, 2009.

[2] Arindam Banerjee, Inderjit S Dhillon, Joydeep Ghosh, and Suvrit Sra. Clustering on the unit hypersphere using von Mises-Fisher distributions. Journal of Machine Learning Research, 6(Sep):1345-1382, 2005.

[3] Tim McGraw, Baba Vemuri, Robert Yezierski, and Thomas Mareci. Segmentation of high angular resolution diffusion MRI modeled as a field of von Mises-Fisher mixtures. In European Conference on Computer Vision, pages 463-475. Springer, 2006.

[4] Abhir Bhalerao and Carl-Fredrik Westin. Hyperspherical von Mises-Fisher mixture (hvmf) modelling of high angular resolution diffusion MRI. In International Conference on Medical Image Computing and Computer-Assisted Intervention, pages 236-243. Springer, 2007.

[5] Ritwik Kumar, Angelos Barmpoutis, Baba C Vemuri, Paul R Carney, and Thomas H Mareci. Multi-fiber reconstruction from DW-MRI using a continuous mixture of von Mises-Fisher distributions. In Computer Vision and Pattern Recognition Workshops, 2008. CVPRW'08. IEEE Computer Society Conference on, pages 1-8. IEEE, 2008.

[6] Fan Zhang, Edwin R Hancock, Casey Goodlett, and Guido Gerig. Probabilistic white matter fiber tracking using particle filtering and von Mises-Fisher sampling. Medical image analysis, 13(1):5-18, 2009.

[7] KJ Painter and T Hillen. Mathematical modelling of glioma growth: the use of diffusion tensor imaging (DTI) data to predict the anisotropic pathways of cancer invasion. Journal of theoretical biology, 323:25-39, 2013.

[8] GK Reynolds, Talia M Nir, Neda Jahanshad, Gautam Prasad, and Paul M Thompson. Using the raw diffusion MRI signal and the von Mises-Fisher distribution for classification of Alzheimer's disease. In Biomedical Imaging (ISBI), 2014 IEEE 11th International Symposium on, pages 1027-1030. IEEE, 2014. 
[9] D. J. Werring, C. A. Clark, G. J. Barker, A. J. Thompson, and D. H. Miller. Diffusion tensor imaging of lesions and normal-appearing white matter in multiple sclerosis. Neurology, 52(8):1626-1626, 1999.

[10] Alexander C Guo, James R MacFall, and James M Provenzale. Multiple sclerosis: diffusion tensor MR imaging for evaluation of normal-appearing white matter 1 . Radiology, 222(3):729-736, 2002.

[11] Yulin Ge, Meng Law, Glyn Johnson, Joseph Herbert, James S Babb, Lois J Mannon, and Robert I Grossman. Preferential occult injury of corpus callosum in multiple sclerosis measured by diffusion tensor imaging. Journal of Magnetic Resonance Imaging, 20(1):1-7, 2004.

[12] SM Hesseltine, M Law, J Babb, M Rad, S Lopez, Y Ge, G Johnson, and RI Grossman. Diffusion tensor imaging in multiple sclerosis: assessment of regional differences in the axial plane within normal-appearing cervical spinal cord. American Journal of Neuroradiology, 27(6):1189-1193, 2006.

[13] Ni Shu, Yaou Liu, Kuncheng Li, Yunyun Duan, Jun Wang, Chunshui Yu, Huiqing Dong, Jing Ye, and Yong He. Diffusion tensor tractography reveals disrupted topological efficiency in white matter structural networks in multiple sclerosis. Cerebral Cortex, 21(11):2565-2577, 2011.

[14] Özgür Yaldizli, Matteo Pardini, Varun Sethi, Nils Muhlert, Zheng Liu, Daniel J Tozer, Rebecca S Samson, Claudia AM Wheeler-Kingshott, Tarek A Yousry, David H Miller, et al. Characteristics of lesional and extra-lesional cortical grey matter in relapsing-remitting and secondary progressive multiple sclerosis: A magnetisation transfer and diffusion tensor imaging study. Multiple Sclerosis Journal, 22(2):150-159, 2016.

[15] Safa Elsheikh, Andrew Fish, Roma Chakrabarti, and Diwei Zhou. Cluster analysis of diffusion tensor fields with application to the segmentation of the corpus callosum. Procedia Computer Science, 90:15-21, 2016.

[16] Kurt Hornik and Bettina Grün. movmf: An R package for fitting mixtures of von Mises-Fisher distributions. Journal of Statistical Software, 58(10):1-31, 2014.

[17] Sandra F Witelson. Hand and sex differences in the isthmus and genu of the human corpus callosum. Brain, 112(3):799-835, 1989. 